\title{
A sífilis na gestação e sua influência na morbimortalidade materno-infantil: uma revisão integrativa
}

\author{
Syphilis in pregnancy and their influence on fetal and maternal morbidity: an integrative review \\ La sífilis en el embarazo y su influencia en la morbilidad y mortalidad materna e infantil: una \\ revisión integradora
}

Recebido: 17/12/2021 | Revisado: 24/12/2021 | Aceito: 04/01/2022 | Publicado: 05/01/2022

Ana Karolyne Monteiro da Silva ORCID: https://orcid.org/0000-0002-5955-9306

Universidade Ceuma, Brasil

E-mail: ana90651@ ceuma.com.br

Amanda Rafaella Garcia Avelino

ORCID: https://orcid.org/0000-0002-4319-4488

Universidade Ceuma, Brasil

E-mail: amandaravelino@gmail.com

Keven Rufino Menezes

ORCID: https://orcid.org/0000-0001-8831-4220

Universidade Ceuma, Brasil

E-mail: kevi.imp@gmail.com

Rafael Abutrab Souza Ramos Silva

ORCID: https://orcid.org/0000-0002-7893-6613 Universidade Ceuma, Brasil

E-mail: rafaelabutrab2014@outlook.com

Ricardo Freitas de Oliveira

ORCID: https://orcid.org/0000-0001-9958-1500 Universidade Ceuma, Brasil

E-mail: ricardo_freitasoliver@hotmail.com

Janine Silva Ribeiro Godoy

ORCID: https://orcid.org/0000-0001-5587-0896

Universidade Ceuma, Brasil

E-mail: janine.silva@ceuma.br

\begin{abstract}
Resumo
Objetivando identificar os fatores base na prevenção da sífilis gestacional e suas complicações materno-fetais, foi realizado uma revisão integrativa de literatura. Utilizando-se as bases de dados SciELO e LILACS, foram selecionados 15 artigos publicados entre o período de 2006 a 2020, bem como os dados obtidos após a realização de uma intervenção acerca do tema junto as gestantes da UBS do bairro Santa Rita, no Município de Imperatriz-MA. Os fatores base na prevenção da sífilis gestacional incluem medidas de profilaxia da doença, adesão adequada ao prénatal para diagnóstico precoce, instituição de tratamento específico, concordância da gestante à terapêutica e acompanhamento por equipe de saúde multiprofissional após o quadro. A promoção de conhecimento sobre a sífilis gestacional e suas complicações tanto para as gestantes quanto para os profissionais de saúde que avaliam e tratam esse grupo é fundamental para a prevenção dessa doença e seus agravos.
\end{abstract}

Palavras-chave: Sífilis; Complicações; Prevenção; Tratamento.

\begin{abstract}
Aiming to identify the basic factors in the prevention of gestational syphilis and its maternal-fetal complications, an integrative literature review was carried out. Using the SciELO and LILACS databases, 22 articles published between the period 2006 to 2020 were selected, as well the data obtained after an intervention on the topic with pregnant women at the UBS in the Santa Rita neighborhood, in the city. of Imperatriz-MA. The basic factors in the prevention of gestational syphilis include measures to prevent the disease, adequate adherence to prenatal care for early diagnosis, institution of specific treatment, agreement of the pregnant woman to therapy and follow-up by a multidisciplinary health team after the condition. The promotion of knowledge about gestational syphilis and its complications both for pregnant women and for health professionals who assess and treat this group is essential for the prevention of this disease and its problems.
\end{abstract}

Keywords: Syphilis; Complications; Prevention; Treatment. 


\begin{abstract}
Resumen
Con el objetivo de identificar los factores básicos en la prevención de la sífilis gestacional y sus complicaciones materno-fetales, se realizó una revisión integradora de la literatura. Utilizando las bases de datos SciELO y LILACS, se seleccionaron 15 artículos publicados entre el período 2006 a 2020, así como los datos obtenidos luego de una intervención sobre el tema con gestantes en la UBS del barrio Santa Rita, en la ciudad de Emperatriz-MA. Los factores básicos en la prevención de la sífilis gestacional incluyen las medidas de prevención de la enfermedad, la adecuada adherencia a la atención prenatal para el diagnóstico precoz, la institución de un tratamiento específico, la aceptación de la gestante a la terapia y el seguimiento por un equipo multidisciplinario de salud después de la afección. La promoción del conocimiento sobre la sífilis gestacional y sus complicaciones tanto a las mujeres embarazadas como a los profesionales de la salud que evalúan y tratan a este grupo es fundamental para la prevención de esta enfermedad y sus problemas.
\end{abstract}

Palabras clave: Sífilis; Complicaciones; Prevención; Tratamiento.

\title{
1. Introdução
}

A Sífilis é uma doença causada pela bactéria espiroqueta treponema pallidum, que pode ser transmitidapor via sexual e materno-fetal/ vertical, que são vias diretas, ou pode ser por via indireta através de objetos e contaminação sanguínea. Não obstante, a sífilis ainda pode ser dividida em adquirida e congênita, a adquirida é caracterizada quanto ao tempo de evolução da doença, onde pode ser latente, primária, secundária ou terciária, enquanto a congênita se configura em recente, quando surge até $\mathrm{o} 2^{\circ}$ ano de vida, e tardia quando ocorre após o segundo ano de vida (Silva, et al, 2020).

A sífilis por infecção fetal ocorre principalmente entre a $16^{\mathrm{a}}$ e $28^{\mathrm{a}}$ semana de gestação, no qual está relacionada diretamente com o índice de não tratamento das gestantes, dessa forma, se a gestante não realizar tratamento corretamente, há uma proporcionalidade com o aumento das chances de ocorrer a sífilis congênita, gerando as consequências referentes ao quadro da doença (Silva, et al, 2020).

A sífilis congênita é uma doença prevensível, desde que a gestante que estiver em risco ou com infecção propriamente dita, receba diagnóstico e tratamento, de forma rápida e efetiva. Dessa forma, também é necessário realizar o rastreio do parceiro ou parceiros sexuais, para apontar para um risco para a gestante de adquirir infecção ou mesmo após diagnóstico, realizar o tratamento do parceiro, para não se ter possíveis complicações posteriormente, assim, se faz necessário um pré-natal coerente para realizar abordagem no âmbito da sífilis, fazendo uma triagem efetiva (Nonato, et al, 2015).

As manifestações clínicas da sífilis congênita são variadas, e podem até mesmo se apresentar de forma assintomática ao nascimento, ou se sintomática, se configura em precoce e tardia, na precoce as manifestações mais comum são: hepatoesplenomegalia, icterícia, pênfigo sifilítico (principalmente palmo-plantar), anormalidades esqueléticas, dentre outras, enquanto na tardia, se relacionam com inflamação cicatricial da infecção precoce, onde pode se apresentar com gomas sifilíticas em diversos tecidos, ceratite intersticial, articulações de Clutton, dentre outras deformidades, que podem ocorrer com a evolução da doença (Domingues, et al, 2013).

O processo de não adesão ou não realização do pré-natal, o aumento da incidência de gravidez na adolescência, o abuso e uso indiscriminado de drogas ilícitas pela gestante ou pelo parceiro, q ausência de parceiro sexual fixo e/ou a coexistência de múltiplos parceiros, baixo nível socioeconômico e precária escolaridade, multiparidade, zona de moradia distantes dos acessos aos serviços de saúde, presença de outras infecções sexualmente transmissíveis (IST's) na mulher ou no parceiro são fatores de risco diretamente ligados à infecção congênita, sendo necessário uma anamnese coerente no período de pré-natal, junto com a triagem sorológica, pois um acompanhamento bem feito reduz os riscos de complicação (Damasceno, 2014).

Desse modo, se faz necessário a realização de ações de educação em saúde, com esclarecimento para a população envolvida, vislumbrando a importância de um acompanhamento pré-natal efetivo para as gestantes ou aquelas que possuem planejamento familiar próximo, buscando contato direto por meio de rodas de conversas, onde se deve discutir as principais prevenções, a importância das consultas, o risco que elas podem se colocar, o uso de determinadas medicações e sobre 
infecções sexualmente transmissíveis, focando também nas complicações em que os seus filhos (em gestação) podem ser expostos, buscando assim uma maior atenção e comprometimento dessa população abordada (Damasceno, 2014).

\section{Metodologia}

Trata-se de um estudo tipo Revisão Integrativa da Literatura, uma vez que esta propõe a discussão não apenas dos resultados obtidos nos textos estudados, mas também de suas fontes, objetivos e resultados, permitindo um olhar amplo sobre o tema, mantendo-se os padrões de clareza, rigor e replicação dos estudos primários. Sendo assim, questiona-se "Por que a sífilis gestacional é um problema de saúde pública no Brasil?"

Foram escolhidas três bases de dados online para a realização da pesquisa na Biblioteca Virtual da Saúde (BVS): LILACS (Literatura Latino-americana e do Caribe de Informação em Ciências da Saúde), MedLine (Medical Literature Analysis and Retrieval System Online) e no SciELO (Scientific Eletronic Library Online). Os descritores foram aplicados de forma combinada, empregando o operador booleano AND: "Transmissão Vertical de Doença Infecciosa AND Sífilis", “Transmissão Vertical de Doença Infecciosa AND Gravidez" e "Sífilis AND Gravidez", envolvendo as bases de dados LILACS e MEDLINE, no idioma português, uma vez que o intuito foi analisar a literatura brasileira acerca do tema. Como critérios de inclusão levou-se em consideração os artigos publicados nos idiomas inglês e português, entre os anos de 2006 e 2020, disponíveis online (gratuitos), com texto completo e que contemplaram a pergunta norteadora deste estudo.

Foram excluídos os artigos que não atenderam aos objetivos pré-estabelecidos ou que estavam no formato de editoriais, cartas ao editor ou opiniões de especialistas, bem como os artigos que extrapolem a janela de tempo proposta, e livros. As buscas foram executadas por dois revisores, de modo independente, a fim de evitar distorções nos resultados. A análise de dados foi realizada por meio da leitura exploratória e seletiva dos artigos encontrados. Após a seleção dos artigos mediante os critérios pré-estabelecidos, foram selecionados 15 artigos e realizado o registro das informações obtidas (título, autor/ano/país, revista e delineamento de pesquisa), sendo os artigos categorizados por meio de um quadro sinóptico.

\section{Resultados}

Em seguida, após a leitura dos artigos completos foram selecionados 15 artigos. Apresenta-se a seguir um fluxograma do processo de seleção dos artigos para revisão (Figura 1). 
Figura 1. Fluxograma do processo de seleção dos artigos para revisão.
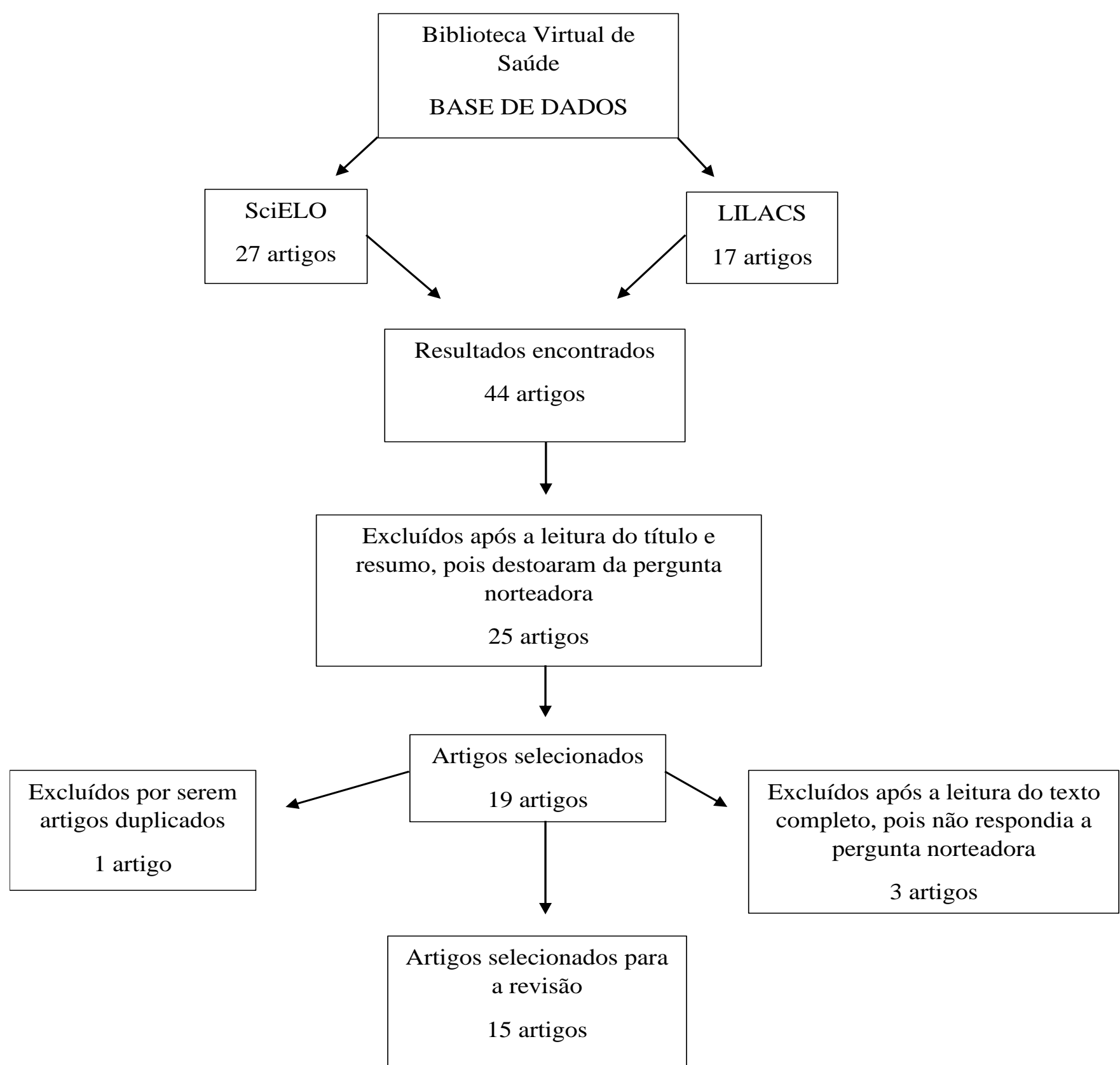

Fonte: Autores.

A partir dos estudos das referêcias selecionadas (Quadro 2) notou-se alguns pontos primordiais relacionados a sífilis gestacional e suas complicações materno-fetais como as medidas de prevenção da doença, a realização de um pré-natal adequado para as gestantes, o diagnóstico precoce da doença e a adesão e acompanhamento correto do tratamento específico.

Com a intervenção proposta para as gestantes, observou-se que existe espaço para o aumento do conhecimento sobre sífilis para esse grupo tendo em vista que muitas gestantes podem ser agentes ativos na disseminação de conhecimentos e práticas úteis na prevenção da doença, bem como compreenderem melhor a necessidade de uma maior adesão ao pré-natal. 
Ademais, notou-se que os profissionais de saúde devem estar capacitados para oferecer as melhores orientações e condutas em todos os âmbitos dessa temática.

Quadro 2 - Sumarização dos artigos encontrados que abordam o tema sífilis gestacional.

\begin{tabular}{|c|c|c|}
\hline Artigo & Autor/Ano/País & Revista \\
\hline $\begin{array}{l}\text { O conhecimento de gestantes com diagnóstico de sífilis } \\
\text { sobre a doença }\end{array}$ & Costa et al, 2016 (Brasil) & Revista interdisciplinar \\
\hline $\begin{array}{l}\text { Construção e validação de uma tecnologia educacional } \\
\text { para prevenção da sífilis congênita }\end{array}$ & Costa et al, 2020 (Brasil) & Acta Paulista de Enfermagem \\
\hline Sífilis secundária em gestante: relato de caso & Wastner et al, 2017 (Brasil) & Archives of Health Investigation \\
\hline Sífilis na gravidez & Damasceno et al, 2014 (Brasil) & Revista Hospital Universitário Pedro Ernesto \\
\hline $\begin{array}{l}\text { Sífilis en la gestante y congénita: perfil epidemiológico } \\
\text { y prevalencia }\end{array}$ & Da Silva et al, 2020 (Brasil) & Enfermería Global \\
\hline $\begin{array}{l}\text { Protocolo Brasileiro para Infecções Sexualmente } \\
\text { Transmissíveis 2020: sífilis congênita e criança exposta } \\
\text { à sífilis }\end{array}$ & Domingues et al, 2021 (Brasil) & Epidemiologia e Serviços de Saúde \\
\hline $\begin{array}{l}\text { Sífilis congênita: evento sentinela da qualidade da } \\
\text { assistência pré-natal }\end{array}$ & Domingues et al, 2013 (Brasil) & Revista Saude Publica \\
\hline Sífilis em gestantes e sífilis congênita no Maranhão & Guimarães et al, 2018 (Brasil) & Arquivos de Ciências da Saúde \\
\hline $\begin{array}{l}\text { Intervenção educacional na Atenção Básica para } \\
\text { prevenção da sífilis congênita }\end{array}$ & Lazarini, 2017 (Brasil) & Revista Latino-Americana de Enfermagem \\
\hline Sífilis materna e congênita: ainda um desafio & Magalhães et al, 2013 (Brasil) & Cadernos de Saúde Pública \\
\hline $\begin{array}{l}\text { Análise dos casos de sífilis congênita em Sobral: } \\
\text { Contribuições para assistência Pré-natal }\end{array}$ & Mesquita et al, 2020 (Brasil) & DST - J. Bras Doenças Sex Transm \\
\hline $\begin{array}{l}\text { Sífilis na gestação e fatores associados à sífilis } \\
\text { congênita em Belo Horizonte-MG, 2010-2013 }\end{array}$ & Nonato, 2015 (Brasil) & Epidemiologia e Serviços de Saúde \\
\hline Abordagens sobre sífilis congênita & Rocha et al, 2020 (Brasil) & Research, Society and Development \\
\hline Sífilis materna e congênita: ainda um desafio & Santos, 2013 (Brasil) & Cad Saúde Pública \\
\hline Sífilis congênita: uma revisão de literatura & Sonda et al, 2013 (Brasil) & Revista de Epidemiologia e Controle de Infecção \\
\hline
\end{tabular}

Fonte: Autores.

\section{Discussão}

A sífilis gestacional ou sífilis congênita (SC) é fruto da disseminação vertical do Treponema pallidum, transmitido da mãe para o filho, que ocorre em qualquer fase da gestação, com probabilidades de 50\% a 100\% na sífilis primária e secundária, $40 \%$ na sífilis latente precoce e $10 \%$ na sífilis latente tardia. É possível transmissão direta no canal do parto. Tal infecção confere riscos para mãe e para o feto, que sem o tratamento adequado, desenvolvem consequências precoces e tardias (Silva $e t$ al., 2019; Sonda et al., 2013).

Ademais, é classificada em dois períodos de diferentes características relacionadas as consequências da doença em: precoce (até dois anos de idade), na qual os sinais e sintomas comuns variam de prematuridade, baixo peso, coriza sanguinolenta, obstrução nasal, osteocondrite, choro ao manuseio, pênfigo palmo plantar, hepatoesplenomegalia, anemia severa, paralisia de membros; e a tardia (com surgimento após os dois anos de idade), e quadro que se estende a mudanças teciduais crônicas como, tíbia em "lâmina de sabre", fronte olímpica, nariz em sela, dentes deformados (dentes de hutchinson), mandíbula curta, surdez neurológica, entre outros (Rocha et al., 2020; Brasil, 2021). 
A Organização Mundial da Saúde (OMS), em 2016, elencou que cerca de 1 milhão de gestantes estavam infectadas com sífilis, promovendo crianças com manifestações clínicas precoces e tardias da Sífilis Congênita. Nas Américas, a taxa de incidência de Sífilis Congênita vem crescendo ao longo dos anos, e os casos notificados no Brasil contribuíram consideravelmente para esse aumento. No Brasil, somente em 2018, foram notificados 158.051 casos de sífilis adquirida, 62.599 casos de sífilis em gestantes, e 26.219 de SC. A inclusão da sífilis gestacional como infecção sexualmente transmissível (IST) de notificação compulsória justifica-se por sua elevada taxa de transmissão vertical, que varia de 30 a $100 \%$ sem o tratamento ou com tratamento inadequado e elevada taxa de prevalência (Brasil, 2008).

Diagnostica-se através dos sinais clínicos e laboratoriais da doença, histórico de infecções e tratamentos prévios, e investigação de exposições recentes. No arsenal diagnóstico, os testes são divididos em dois grupos: diretos e imunológicos. Os exames diretos incluem a pesquisa de Treponema pallidum em amostras coletadas de lesões, utilizando microscopia de campo escuro, impregnação pela prata, imunofluorescência ou técnicas de biologia molecular por reação de cadeia da polimerase. Os testes imunológicos, testes treponêmicos (TT) e testes não treponêmicos (TNT), são os mais utilizados e caracterizam-se pela pesquisa de anticorpos em amostras de sangue total, soro, plasma ou líquor (Figueiredo et al., 2020).

Dessa forma, a utilização de testes rápidos apresenta vantagens quanto à precocidade do diagnóstico e otimização do tempo, tendo início do tratamento imediato, na mesma consulta. As gestantes com testes rápidos reagentes para sífilis deverão ser consideradas como portadoras de sífilis até que se prove o contrário. São testes amplamente utilizados nos laboratórios, de baixo custo e caracterizam-se por terem resultados semi-quantitativos, pois nos casos de resultados reagentes, realiza-se a diluição da amostra para titulação desses anticorpos e emissão do resultado. Os testes não treponêmicos detectam anticorpos IgM e IgG anticardiolipina não específicos para T. pallidum. A cardiolipina consiste no material liberado pelas células humanas danificadas em decorrência da sífilis, e também pelo treponema durante a sua destruição no organismo (Luo et al., 2021).

Assim, o principal exame utilizado para investigação da doença é a sorologia não-treponêmica (VDRL), sendo indicada tanto no diagnóstico como acompanhamento da terapêutica. O VDRL (do inglês Venereal Disease Research Laboratory) baseia-se no uso de uma suspensão antigênica composta por uma solução alcoólica contendo cardiolipina, colesterol e lecitina purificada e utiliza soro inativado como amostra (Brasil, 2021).

Devem ser consideradas reagentes as amostras que apresentarem reatividade em qualquer uma das diluições, incluindo aquelas reativas somente com a amostra pura (diluição 1:1). Os passos de diluição de uma amostra normalmente são executados com fator 2 de diluição. Ou seja, o título 1 (diluição 1:1) significa que a amostra foi analisada pura, isto é, a amostra foi testada sem diluição; O título 2 (diluição 1:2) significa que um volume de amostra foi diluído em um mesmo volume de solução tampão; 1:4 significa que um volume da amostra foi diluída em solução tampão em três vezes o volume de amostra, e assim por diante. Dessa forma, uma amostra com reatividade até diluição 1:256 possui mais anticorpos do que uma amostra com reatividade até diluição 1:2 (Brasil, 2020).

Logo, para o monitoramento da resposta ao tratamento, onde se avalia se a titulação da amostra caiu ou aumentou em relação a última testagem do paciente, recomenda-se a utilização do mesmo tipo de teste não treponêmico, preferencialmente do mesmo fabricante, desde o diagnóstico até o último teste de monitoramento, para que possa haver a correta comparação dos títulos obtidos. Além disso, somente deve ser considerado significativo para conduta clínica o aumento ou a diminuição do título em pelo menos duas diluições, devido a possibilidade de variação do resultado, intrínseca da metodologia, em até uma diluição (Organização Mundial da Saúde, 2016).

Considera-se caso de sífilis na gestação: toda gestante com evidência clínica de sífilis e/ou com sorologia não treponêmica reagente, com qualquer titulagem, mesmo na ausência de resultado de teste treponêmico, realizada no pré-natal ou no momento do parto ou curetagem. Deverá ser oferecido teste não treponêmico (VDRL) na primeira consulta de pré-natal 
para todas as gestantes, idealmente no primeiro trimestre de gestação e no início do terceiro trimestre. Na maternidade, realizase VDRL em toda mulher admitida para parto (nascido vivo ou natimorto) ou curetagem (após aborto). Nos casos de mães com VDRL reagente na gestação ou no parto, colhe-se o sangue periférico do recém-nascido para realização de teste não treponêmico, além de proceder à avaliação geral da criança com a solicitação dos exames de líquor, raio-X de ossos longos e hemograma (Brasil, 2020).

Outrossim, o tratamento da sífilis congênita dependerá de quatro fatores: identificação de sífilis na mãe, adequação ao tratamento, presença de evidências clínicas, laboratoriais e radiológicas no neonato e comparação da sorologia nãotreponêmica materna e do neonato (Domingues et al., 2021).

Indica-se o tratamento com penicilina G cristalina na dose de $50.000 \mathrm{UI} / \mathrm{Kg}$, por via endovenosa, de $12 \mathrm{em} 12$ horas nos primeiros 7 dias de vida e de 8 em 8 horas até completar 10 dias de vida, ou por penicilina G procaína 50.000 UI/Kg, dose única diária, por via intramuscular durante 10 dias nas seguintes situações: recém-nascido sintomático, com alteração clínica, sorológica, radiológica e/ou hematológica, cuja mãe com sífilis foi adequadamente tratada, ou cuja mãe com sífilis não foi tratada ou foi inadequadamente tratada (tratamento realizado com penicilina antes de 30 dias do parto, ou sem penicilina) e em recém-nascido de mães tratadas, que possuem VDRL maior que o materno, ou menor ou igual ao materno sem possibilidades de seguimento clínico, ambos os casos com alterações nos exames. Caso haja alterações no líquor, em todos os casos, utiliza-se somente o esquema de penicilina G cristalina (Domingues et al., 2021; Brasil, 2020).

$\mathrm{O}$ tratamento com penicilina G benzatina, $50.000 \mathrm{UI} / \mathrm{Kg}$, dose única, intramuscular, é indicada para: recém-nascidos assintomáticos de mães incorretamente tratadas, ou de mães tratadas sem possibilidade de acompanhamento clínico, ambos os casos com exames e VDRL negativos; e em recém-nascidos, de mães tratadas, que possuem VDRL menor ou igual ao materno, com outros exames negativos (Domingues et al., 2021; Brasil, 2020).

$\mathrm{O}$ tratamento com penicilina $\mathrm{G}$ cristalina, de $4 \mathrm{em} 4$ horas, é feito em crianças com mais de 1 mês de vida, com quadro clínico e sorológico compatível com sífilis congênita. Em qualquer tratamento, caso haja interrupção por 1 dia, este deverá ser reiniciado (Domingues et al., 2021; Brasil, 2020).

No que tange à prevenção, tendo a sífilis como IST que é facilmente diagnosticada pelo VDRL e com eficácia tratada pela penicilina, os estudos mostram que a não realização do pré-natal é considerada como um dos principais fatores responsáveis pelos casos de sífilis congênita. A falta de realização de exames para o diagnóstico da sífilis; dificuldade em reconhecer os sinais da doença na mãe; falhas na interpretação dos resultados de testes sorológicos e falhas ou ausência de tratamento da mãe e/ou do parceiro são fatores relacionados ao pré-natal inadequado. A assistência pré-natal estendida a todas as grávidas seria a maneira mais lógica de se eliminar a sífilis materna e suas consequências. Observa-se a necessidade de maiores esclarecimentos às grávidas sobre a gravidade e o modo de transmissão da sífilis e de suas consequências para o concepto, sem esquecer de instituir os métodos de proteção como uso de preservativos masculinos e femininos como meio eficiente na proteção da gestante e do bebê (Vilela et al., 2019; Souza et al., 2018).

\section{Conclusão}

Diante das complicações relacionadas à sífilis durante o período de gestação, se faz necessário assegurar uma prática de assistência à esse grupo, vislumbrando um maior esclarecimento sobre o assunto, visto que diante desse estudo, foi analisado que há uma carência de conhecimento acerca da infecção por sífilis e os meios de diagnosticar e tratamento, e principalmente os meios de prevenção, não obstante, deve-se realizar uma política pública de intervenção no bairro Santa Rita, uma bairro no qual possui uma grande demanda e desses forma, um maior contato através de palestras e o uso de materiais visuais como panfletos e folders em geral, são fundamentais para efetivar uma educação em saúde acerca dessa doença, e assim buscar prevenir e reduzir o índice de incidência e prevalência, dando ênfase nos fatores de risco no qual essa população está 
vulnerável. São medidas cabíveis para os profissionais da saúde, ou os acadêmicos também, em conjunto com os órgãos públicos.

Portanto, diante desse cenário, é imperativo que o estudo aponta para uma necessidade intervencionista nessa área, para que haja retornos positivos no âmbito da saúde pública e geral, não apenas no bairro Santa Rita, mas a cidade de ImperatrizMA como um todo, pode chegar a ser beneficiada com tais ações.

\section{Referências}

Brasil (2008). Fundação Nacional de Saúde (Funasa). Diretrizes de educação em saúde visando à promoção da saúde: documento base - documento I: Brasília. Brasil (2006). Ministério da Saúde (MS). Secretaria de Vigilância em Saúde. Programa Nacional de DST/AIDS. Diretrizes para controle da sífilis congênita: manual de bolso. (2a ed.),MS.

Brasil (2021). Boletim Epidemiológico. Secretaria de Vigilância em Saúde Ministério da Saúde. Editora MS/CGDI.

Cabral, B. T. V., da Costa Dantas, J., da Silva, J. A., \& de Oliveira, D. A. (2017). Sífilis em gestante e sífilis congênita: um estudo retrospectivo. Revista ciência plural, 3(3), 32-44.

Costa, J. S., et al (2016). O conhecimento de gestantes com diagnóstico de sífilis sobre a doença. Revista interdisciplinar, v. 9, n. 2, p. 79-89.

Costa, C. C. D., Gomes, L. F. D. S., Teles, L. M. R., Mendes, I. C., Oriá, M. O. B., \& Damascen, A. K. D. C. (2020). Construção e validação de uma tecnologia educacional para prevenção da sífilis congênita. Acta Paulista de Enfermagem, 33.

da Fonseca Wastner, B., Melzer, R. S., do Nascimento, T. C. D. L., Parise, G. K., Stramandinoli-Zanicotti, R. T., \& Sassi, L. M. (2017). Sífilis secundária em gestante: relato de caso. Archives Of Health Investigation, 6(9).

Damasceno, A. B., Monteiro, D. L., Rodrigues, L. B., Barmpas, D. B. S., Cerqueira, L. R., \& Trajano, A. J. (2014). Sífilis na gravidez. Revista Hospital Universitário Pedro Ernesto, 13(3).

de Almeida Lemos, L. S. C., de Souza, G. S., Vasconcelos, B. M., Gama, C. R., de Medeiros Silva, L. S., Cerqueira, T. M. G., ... \& da Silva Noberto, D. (2019). O pré-natal como ferramenta na prevenção da sífilis congênita: uma revisão integrativa da literatura. Brazilian Journal of Health Review, 2(3), 16161623 .

Domingues, R. M. S. M., Saracen, V., Hartz, Z. M. D. A., \& Leal, M. D. C. (2013). Sífilis congénita: evento centinela de la calidad de la asistencia prenatal. Revista de Saúde pública, 47(1), 147-157.

Epidemiológica, S. D. V. (2008). Sífilis congênita e sífilis na gestação. Revista Saúde Pública, 42(4), 768-72.

Figueiredo, D. C. M. M. D., Figueiredo, A. M. D., Souza, T. K. B. D., Tavares, G., \& Vianna, R. P. D. T. (2020). Relação entre oferta de diagnóstico e tratamento da sífilis na atenção básica sobre a incidência de sífilis gestacional e congênita. Cadernos de Saúde Pública, 36.

Freitas Silva, M. F. C., Pereira, S. M. X., Aidar, T. D. P. S., de Souza, R. G., de Carvalho Costa, R. F., de Oliveira, L. A. G., ... \& Dantas, P. C. (2020). Sífilis congênita como uma abordagem sistêmica. Brazilian Journal of Development, 6(7), 51840-51848.

Guimarães, T. A., Alencar, L. C. R., Fonseca, L. M. B., Gonçalves, M. M. C., \& da Silva, M. P. (2018). Sífilis em gestantes e sífilis congênita no Maranhão. Arquivos de Ciências da Saúde, 25(2), 24-30.

Lazarini, FM, \& Barbosa, DA (2017). Intervenção educacional na Atenção Básica para prevenção da sífilis congênita1. Revista Latino-Americana de Enfermagem, 25.

Luo, Y., Xie, Y., \& Xiao, Y. (2021). Ferramentas de diagnóstico laboratorial para sífilis: situação atual e perspectivas futuras. Fronteiras em microbiologia celular e de infecção, 896.

Magalhães, D. M. D. S., Kawaguchi, I. A. L., Dias, A., \& Calderon, I. D. M. P. (2013). Sífilis materna e congênita: ainda um desafio. Cadernos de Saúde Pública, 29, 1109-1120.

Mesquita, K. O., Lima, G. K., Filgueira, A. A., Flôr, S. M. C., Freitas, C. A. S. L., Linhares, M. S. C., \& Gubert, F. A. (2012). Análise dos casos de sífilis congênita em Sobral, Ceará: contribuições para assistência pré-natal. DST-J Bras Doenças Sex Transm, 24(1), 20-7.

Milanez, H., \& Amaral, E. (2008). Por que ainda não conseguimos controlar o problema da sífilis em gestantes e recém-nascidos?. Revista Brasileira de Ginecologia e Obstetrícia, 30(7), 325-327.

Netto, J. L. D. M. G., da Silva, A. P., Medeiros, E. B., Rodrigue, M. M. D., de Carvalho, A. K. F., \& de Lima Soares, V. (2021). Sífilis em gestantes no nordeste do Brasil: aspectos epidemiológicos no período de 2010 E 2019. The Brazilian Journal of Infectious Diseases, $25,101324$.

Nonato, S. M., Melo, A. P. S., \& Guimarães, M. D. C (2015). Sífilis na gestação e fatores associados à sífilis congênita em Belo Horizonte-MG, 20102013. Epidemiologia e Serviços de Saúde, 24., 681-694.

Rocha, C. C, Lima, T. S, Silva, R. A. N, \& Abrão, R. K. (2020). Abordagens sobre sífilis congênita. Research, Society and Development, 9 (8), e984986820e984986820. 
Research, Society and Development, v. 11, n. 1, e24511124891, 2022

(CC BY 4.0) | ISSN 2525-3409 | DOI: http://dx.doi.org/10.33448/rsd-v11i1.24891

Rocha, A. F. B., Araújo, M. A. L., Barros, V. L. D., Américo, C. F., \& Silva Júnior, G. B. D. (2021). Complicações, manifestações clínicas da sífilis congênita e problemas relacionados à prevenção: revisão integrativa. Revista Brasileira de Enfermagem, 74.

Silva, J. G., Gomes, G. C., Ribeiro, J. P., de Jung, B. C., de Oliveira Nörberg, P. K., \& Mota, M. S. (2019). Sífilis gestacional: repercussões para a puérpera. Cogitare Enfermagem, 24.

Sousa, F. D. C. A., Lopes, D. S. D. C., da Costa, A. C. M., da Silva, E. B., da Silva, W. C., de Oliveira, E. H., ... \& Andrade, E. W. O. F. (2020). Perfil da Sífilis na Gestação no Período de 2007/2016 em Caxias-MA. Revista Enfermagem Atual In Derme, 91(29).

Sonda, E. C, Richter, F. F, Boschetti, G., Casasola, M. P, Krumel, C. F, \& Machado, C. .P H (2013). Sífilis congênita: uma revisão da literatura. Revista de Epidemiologia e Controle de Infecção, 3 (1), 28-30.

Organização Mundial da Saúde. (2016). Diretrizes da OMS para o tratamento do Treponema pallidum (sífilis). 